\title{
Enhancing the evaluation of noble gas data with the software PANGA
}

\author{
SOPHIE NEGELE, ANN-KRISTIN KUNZ AND WERNER \\ AESCHBACH \\ Institute of Environmental Physics \\ Presenting Author: sophie.negele@iup.uni-heidelberg.de
}

The evaluation of measured noble gas concentrations in groundwater with respect to different types of excess air or in some cases degassing models yields information about the conditions during recharge, such as the noble gas temperature (NGT) or the amount of excess air (EA). PANGA [1] is a software tool that provides least squares fitting for noble gas concentrations to determine different parameters from the models. It enables both an analytical error propagation and a Monte Carlo analysis of the results. Recently, the software has been updated with new high-precision noble gas solubilities (from [2]). Small but systematic effects on the estimated values of both NGT and EA could be demonstrated and a systematic analysis on the achievable precision of NGT estimates was conducted. The updated software is now ready to be applied to new and existing noble gas data sets. We demonstrate the use of PANGA for a range of different applications.

Firstly, as the unique noble gas signature related to subglacial recharge of groundwater sampled in Estonia is not yet quantitatively understood, the software has been used to investigate various new models and to quantify the goodness of the corresponding fits. Secondly, for a more comprehensive study, a variety of groundwater data from the literature has been analysed, especially with regard to systematics in the amount of EA, which so far has received little attention, although it is known that EA can provide information about hydroclimatic changes back to the last glacial. Thirdly, PANGA also provides the means to re-investigate selected groundwater studies in more detail, especially regarding samples with noble gas patterns that have been found to be difficult to interpret.

[1] Jung and Aeschbach (2018), Environmental Modelling \& Software 103, 120-130.

[2] Jenkins et al. (2019), Marine Chemistry 211, 94-107. 